\title{
ASSESSMENT OF THE GENETIC DIVERSITY IN AEGILOPS TAUSCHII (COSS.) BY USING SSR MARKERS AND MORPHYSIOLOGICAL TRAITS
}

\author{
ABBAS, A. - YU, H. Y. - CUI, H. L. - YU, H. L. - LI, X. J.* \\ Key Laboratory of Weed and Rodent Biology and Management, Institute of Plant Protection, \\ Chinese Academy of Agricultural Sciences \\ *Corresponding author \\ e-mail:xjli@ippcaas.cn; phone: +86-(0)10-6281-3309 \\ (Received $19^{\text {th }}$ Oct 2018; accepted $5^{\text {th }}$ Dec 2018)
}

\begin{abstract}
Aegilops tauschii Coss. $(2 \mathrm{n}=2 \mathrm{x}=14, \mathrm{DD})$ is a problematic weed and has contributed in the D genome of common wheat. The genetic diversity among 40 Chinese populations were evaluated by microsatellites marker and morphysiological traits. Dry weight biomass showed variation while in the case of plant height high variation was found between populations. We determined 27 alleles by using eight primers with an average of 3.37 allele per locus. The maximum polymorphism information content (PIC) was 0.63 with an average of 0.20 and maximum allele frequency was 1.00 with an average of 0.88 . Cluster analysis divided Aegilops tauschii into different groups, which showed obvious genetic difference between these populations. This study will be helpful for weed management and wheat crop breeding program.
\end{abstract}

Keywords: Aegilops tauschii, molecular marker, genetic diversity, morphysiological trait, microsatellites

Abbreviations: PIC: Polymorphism Information Content

\section{Introduction}

(Aegilops tauschii Coss.) (Ae. tauschii) has a troublesome effect on wheat crop growing areas of China (Dudnikov, 2000; Zhang et al., 2007). It is native to tropical Asia to temperate Asia and Europe (Wei et al., 2008). It has infested about 330,000 hectares (Zhang et al., 2007) of winter wheat in different provinces including Shanxi, Shandong, Henan, Shaanxi, Inner Mongolia, Jiangsu and Hebei in China. Moreover, due to insufficient prevention and limitation of control strategies, the spreading pace of this weed tremendously damage winter wheat productivity, particularly in the main wheat producing regions. Ae. tauschii distributed from the Mediterranean region; present in Syria, Russia, Kazakhstan, Afghanistan, Pakistan, Turkey, all the way to Iran and extending to the eastwards of Yili Valley of Xinjiang in China (Li, 2005). Iran and Yili valley of Xinjiang were usually familiar with the center of the origin of Ae. tauschii. Ae. tauschii is recognized in natural habitats for its world distribution, whereas subspecies (strangulate) of Aegilops is native to south-western Caspian Iran and Afghanistan (Ogbonnaya et al., 2005; Wang et al., 2013; Kalia et al., 2016). Some scientists also proposed that Ae. tauschii presented in the Yellow river region in China was introduced with common wheat through trade along the Silk Road (Li and Mo, 2004; Zhao, 2007).

Ae. tauschii involved in the origin of the hexaploid wheat. Moreover, crop breeding has resulted in genetic diversity among the hexaploid wheat and Ae. tauschii populations. On the base of the morphological parameter, decidedly less genetic differentiation in the D genome of the wheat crop exists (Zahra et al., 2010). Due to the 
genetic similarity of Ae. tauschii with wheat, it may have an essential role in wheat crop improvement (Knaggs et al., 2000). Through hybridization, many useful biotic and abiotic stress resistance genes of Ae. tauschii can be utilized in wheat variety improvement programs (Hsam et al., 2001).

Genetic diversity can assessed by phenotypic (physiological and morphological parameters) and microsatellite markers (AFLP, RFLP, and SSR). Morphological parameters of crops are useful for introductory assessment because of their rapid range for diversity (Majidi et al., 2009; Sun et al., 2014). Molecular markers used to evaluate the genetic diversity because of their polymorphism, reproducibility, co-dominance, and simplicity (Roder et al., 1995). In molecular markers, simple sequence repeats (SSR) are universal and commonly used for genetic diversity evaluation (Vieira et al., 2016). Furthermore, SSR markers are more critical for crop improvement (Mian et al., 2005). Aegilops species possessing the D genome could be rich abiotic sources. Molecular markers have been used in several studies to assess genetic diversity among different populations (Roy et al., 2006). Genes from Ae. tauschii can be utilized in wheat variety improvement programs (Hsam et al., 2001).

In China, Ae. tauschii is a problematic weed and competes for resources from the early stage until maturity stage in wheat. It is scattered in more than ten provinces all across the country. Due to morphological similarities with the wheat crop, it is difficult to be controlled with herbicides. It is known that DD genome of Ae. tauschii has a rich source of potential variability. For genetic diversity of Ae. tauschii populations collected from five different provinces of China were assessed by using morphysiological traits and fluorescent dye-labeled SSR markers. The results of this study will be beneficial to both weed management and wheat breeding.

\section{Materials and methods}

Forty populations of Ae. tauschii seeds were used from five different provinces (Henan, Shaanxi, Shandong, Hebei, and Shanxi) of China (Table 1, Fig. 1). The experiment was conducted at the greenhouse of the Institute of Plant Protection, Chinese Academy of Agriculture Sciences, Beijing, China. Each population was replicated four times following a completely randomized design for pot experiment. Plants floated over half-strength Hoagland's nutrient solution (Hoagland and Arnon, 1950). Sixty days after sowing, plant height $(\mathrm{cm})$ and dry weight biomass $(\mathrm{g})$ were recorded. For SSR analysis, plant leaves were taken and stored at $-80^{\circ} \mathrm{C}$ for DNA extraction. DNA was isolated from leaves by plant kit (TIANGEN). DNA purification and quantification were carried out following Shah et al. (2009). SSR primers were designed by BATCHPRIMER3 (Table 2).

Table 1. Aegilops tauschii populations collected from different provinces of China

\begin{tabular}{c|c}
\hline Name of province & Population Collection \\
\hline Henan & 10 \\
Shaanxi & 3 \\
Shandong & 10 \\
Hebei & 12 \\
Shanxi & 5 \\
\hline Total & 40 \\
\hline
\end{tabular}




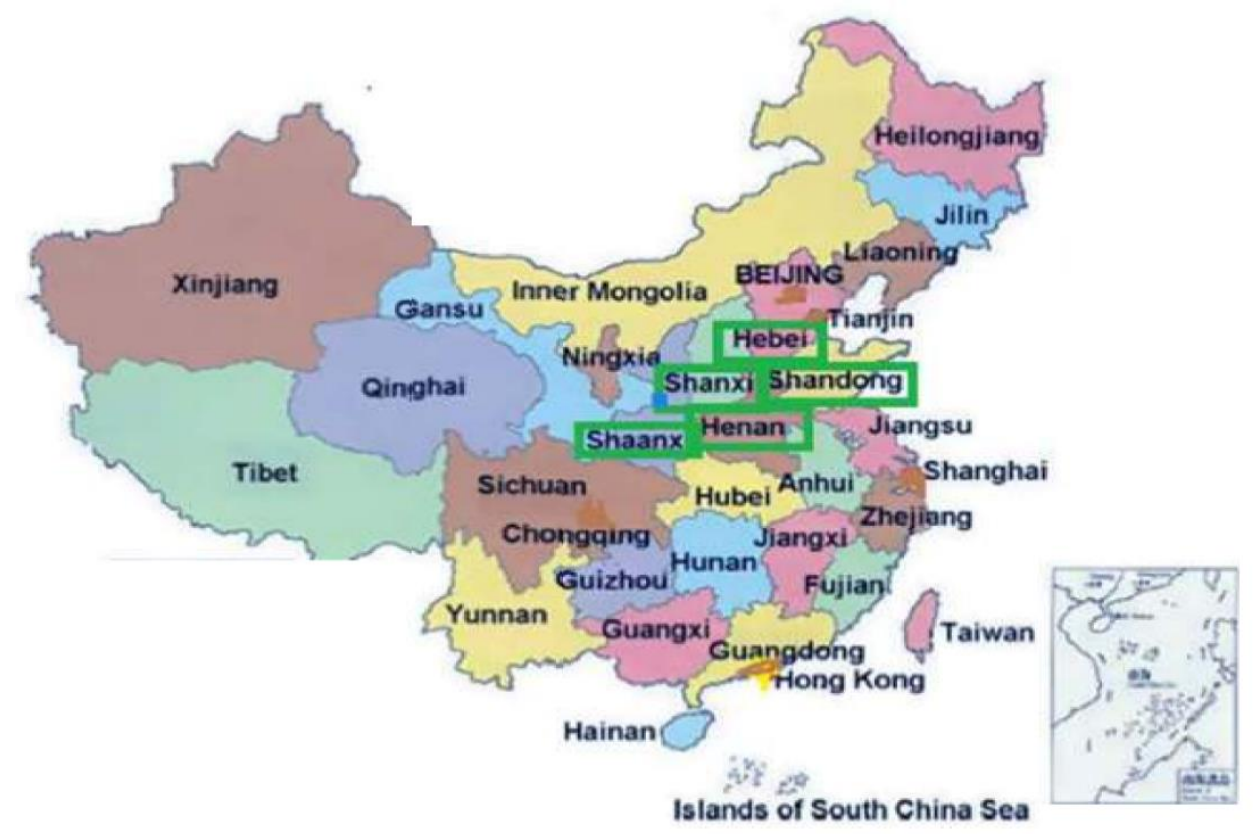

Figure 1. Collection of Aegilops tauschii population's seed from different provinces of China.

Circles indicated provinces for seed collection

Table 2. Details of forward and reverse SSR primers

\begin{tabular}{c|c|c}
\hline Primers & Forward Primer (5'-3') & Reverse Primer (5'-3') \\
\hline AG-5 & AGAACATCTGGCGTAACATAG & GGTTTTGTCGCAGAATTAGTA \\
AG-7 & AGCTTCATACGGCTTCTCTAT & AGCGCTTTTTCTTATTCTAGC \\
AG-12 & TGCAGAAACTACCCAAATCTA & GCCACAAGGGACTATCTAAAC \\
AG-14 & AGAGCAAATATAGGACCCAAG & CTCTCGTATTCGTCCTCTGA \\
AG-15 & ATTATCGCTTAGCTTTCGACT & GTTGCAAAAATAAGAGCTTGA \\
AG-18 & TTGACACGAGGAACTACTCAC & CTGTCTCGCAATACCTTCTAC \\
AG-19 & CTTTGCCACCTACTGCTACTA & CGGATACTGCCATACAATTAC \\
AG-20 & CCAGTTAAGGTGGGATATGAT & GATTGGCGGATTTCTAATAGT \\
\hline
\end{tabular}

Polymerase chain reactions (PCRs) were carried out in $20 \mu \mathrm{L}$ reactions comprising 40 ng of genomic DNA $1 \mu \mathrm{L}, 0.6 \mu \mathrm{M}$ of each forward and reverse primer, $7.8 \mu \mathrm{M}$ of $\mathrm{ddH}_{2} \mathrm{O}$ and $10 \mu \mathrm{M}$ of PCR master mix. The PCR profile consisted of denaturation at $95^{\circ} \mathrm{C}$ for 10 min, followed by 35 cycles of $95^{\circ} \mathrm{C}$ for $30 \mathrm{sec}, 45^{\circ} \mathrm{C}-60^{\circ} \mathrm{C}$ (depending on primers) for 30 sec and $72^{\circ} \mathrm{C}$ for $2 \mathrm{~min}$, with a final extension at $72^{\circ} \mathrm{C}$ for $10 \mathrm{~min}$. The genetic diversity of these populations were analyzed by using eight SSR fluorescent markers by capillary electropherogram.

\section{Statistical analysis}

Means, variances and coefficient of variances for plant height and dry weight biomass were measured by Statistix-8.0. For molecular data, allelic size was determined by using GeneMarker version 2.2.0 (Applied Biosystems). PowerMarker 3.1 was used to determine the allele amplification, allele frequency and polymorphism information content. Phylogenetic tree was drawn by PowerMarker MEGA 3.5. 


\section{Results}

Means, variances and coefficient of variance in 40 Ae. tauschii populations showed variation in dry weight biomass and plant height (Table 3). Different populations have a different coefficient of variance in plant height, with maximum $12 \%$ and minimum $1.8 \%$. In dry weight biomass, maximum and minimum coefficient of variance were recorded $25 \%$ and $3 \%$ in different populations. Analysis of variance in plant height and dry weight biomass showed a relationship between populations, but some populations showed variation as compared with others in these two traits. Populations 6, 9, and 23 showed variation in plant height (Fig. 2), and populations 23 and 26 showed variation in dry weight biomass (Fig. 3). Amplification of eight primers in Ae. tauschii showed 27 alleles with an average of 3.37, maximum polymorphism information content (PIC) was 0.63 with the average of 0.20 , and allele frequency ranges 0.41 to 1 with an average of 0.88 (Table 4). D genome microsatellite markers revealed the allelic range in the Chinese Ae. tauschii populations and has extended and showed polymorphism.

Table 3. Mean standard deviation (STDEV) and coefficient of variance (CV\%) of plant height $(\mathrm{cm})$ and dry weight biomass $(\mathrm{g})$ of 40 Aegilops tauschii populations

\begin{tabular}{|c|c|c|c|c|c|c|}
\hline \multirow{2}{*}{ Populations } & \multicolumn{3}{|c|}{ Plant Height (cm) } & \multicolumn{3}{|c|}{ Dry Weight Biomass (g) } \\
\hline & Mean & STDEV & CV\% & Mean & STDEV & CV\% \\
\hline PI & 11.7 & 0.75 & 8.5 & 3.94 & 0.29 & 7 \\
\hline $\mathrm{P} 2$ & 15.0 & 1.47 & 9.8 & 3.24 & 0.45 & 14 \\
\hline P3 & 17.9 & 1.03 & 5.8 & 3.29 & 0.38 & 11 \\
\hline $\mathrm{P} 4$ & 13.6 & 0.30 & 2.2 & 3.57 & 0.44 & 12 \\
\hline P5 & 14.6 & 0.43 & 2.9 & 3.51 & 0.53 & 15 \\
\hline P6 & 12.1 & 0.45 & 3.8 & 3.00 & 0.49 & 16 \\
\hline P7 & 13.8 & 0.57 & 4.1 & 3.50 & 0.41 & 12 \\
\hline P8 & 13.8 & 0.24 & 1.8 & 3.29 & 0.26 & 8 \\
\hline P9 & 13.9 & 0.63 & 4.5 & 3.87 & 0.12 & 3 \\
\hline $\mathrm{P} 10$ & 14.4 & 0.48 & 3.3 & 4.02 & 0.11 & 3 \\
\hline P11 & 14.3 & 0.65 & 4.5 & 2.85 & 0.25 & 9 \\
\hline P12 & 14.4 & 0.51 & 3.5 & 3.10 & 0.33 & 11 \\
\hline P13 & 13.8 & 0.65 & 4.7 & 2.71 & 0.38 & 14 \\
\hline P14 & 15.8 & 0.73 & 4.6 & 3.14 & 0.42 & 13 \\
\hline P15 & 13.8 & 0.74 & 5.4 & 3.07 & 0.38 & 13 \\
\hline P16 & 12.6 & 0.48 & 3.8 & 2.92 & 0.23 & 8 \\
\hline P17 & 11.6 & 0.75 & 6.5 & 2.91 & 0.16 & 6 \\
\hline P18 & 14.4 & 0.48 & 3.3 & 2.98 & 0.27 & 9 \\
\hline P19 & 10.9 & 1.07 & 9.8 & 2.80 & 0.08 & 3 \\
\hline $\mathrm{P} 20$ & 15.5 & 0.41 & 2.7 & 2.72 & 0.17 & 6 \\
\hline $\mathrm{P} 21$ & 15.3 & 0.39 & 2.6 & 3.38 & 0.31 & 9 \\
\hline $\mathrm{P} 22$ & 12.6 & 0.75 & 5.9 & 3.81 & 0.13 & 4 \\
\hline $\mathrm{P} 23$ & 13.4 & 0.48 & 3.6 & 5.02 & 0.50 & 10 \\
\hline $\mathrm{P} 24$ & 11.4 & 1.37 & 12.0 & 3.89 & 0.21 & 5 \\
\hline $\mathrm{P} 25$ & 11.7 & 0.53 & 4.5 & 3.13 & 0.13 & 4 \\
\hline $\mathrm{P} 26$ & 13.2 & 0.40 & 3.0 & 2.49 & 0.52 & 21 \\
\hline $\mathrm{P} 27$ & 12.6 & 0.43 & 3.5 & 2.69 & 0.30 & 11 \\
\hline P28 & 14.9 & 0.84 & 5.6 & 2.80 & 0.24 & 9 \\
\hline P29 & 13.2 & 0.29 & 2.2 & 3.78 & 0.23 & 6 \\
\hline P30 & 14.2 & 0.86 & 6.0 & 3.56 & 0.31 & 9 \\
\hline P31 & 14.9 & 0.52 & 3.5 & 3.91 & 0.38 & 10 \\
\hline P32 & 12.5 & 0.71 & 5.7 & 3.84 & 0.10 & 3 \\
\hline P33 & 13.1 & 0.59 & 4.5 & 3.82 & 0.66 & 17 \\
\hline P34 & 12.3 & 0.86 & 7.0 & 3.00 & 0.35 & 12 \\
\hline P35 & 15.4 & 0.48 & 3.1 & 3.46 & 0.88 & 25 \\
\hline P36 & 14.4 & 0.48 & 3.3 & 2.79 & 0.39 & 14 \\
\hline P37 & 14.2 & 0.26 & 1.8 & 3.17 & 0.46 & 15 \\
\hline P38 & 14.6 & 0.42 & 2.9 & 3.20 & 0.26 & 8 \\
\hline P39 & 12.2 & 0.93 & 7.6 & 2.94 & 0.34 & 12 \\
\hline $\mathrm{P} 40$ & 15.0 & 1.42 & 9.4 & 3.10 & 0.26 & 9 \\
\hline
\end{tabular}




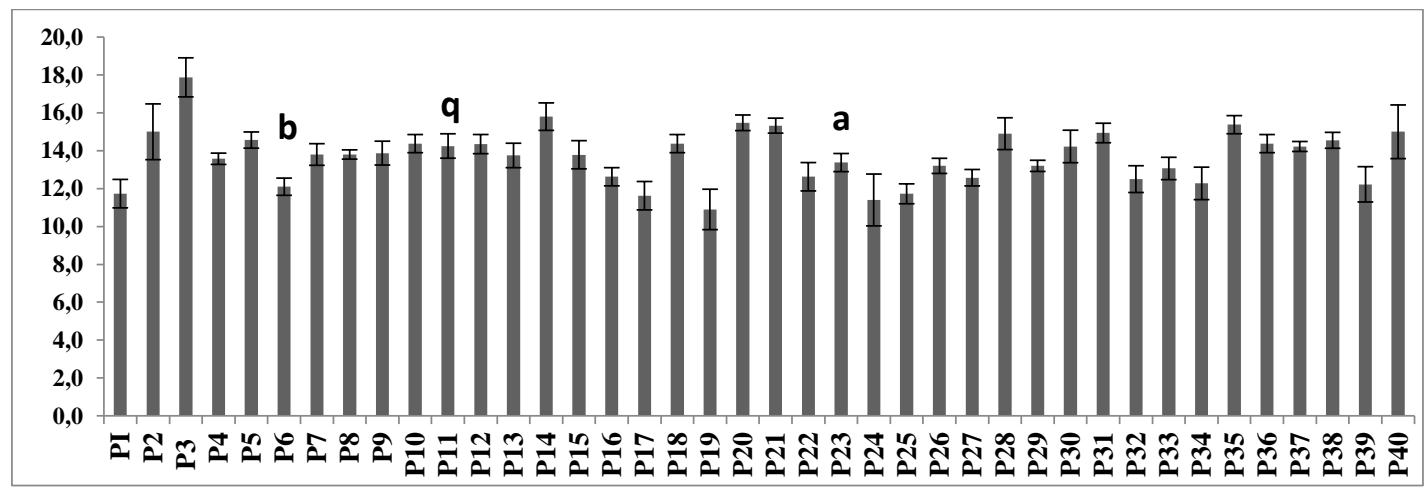

Figure 2. Mean value of plant height of 40 populations of Aegilops tauschii. Bar indicating with letters showed variation. While other populations showed a close relationship with each other

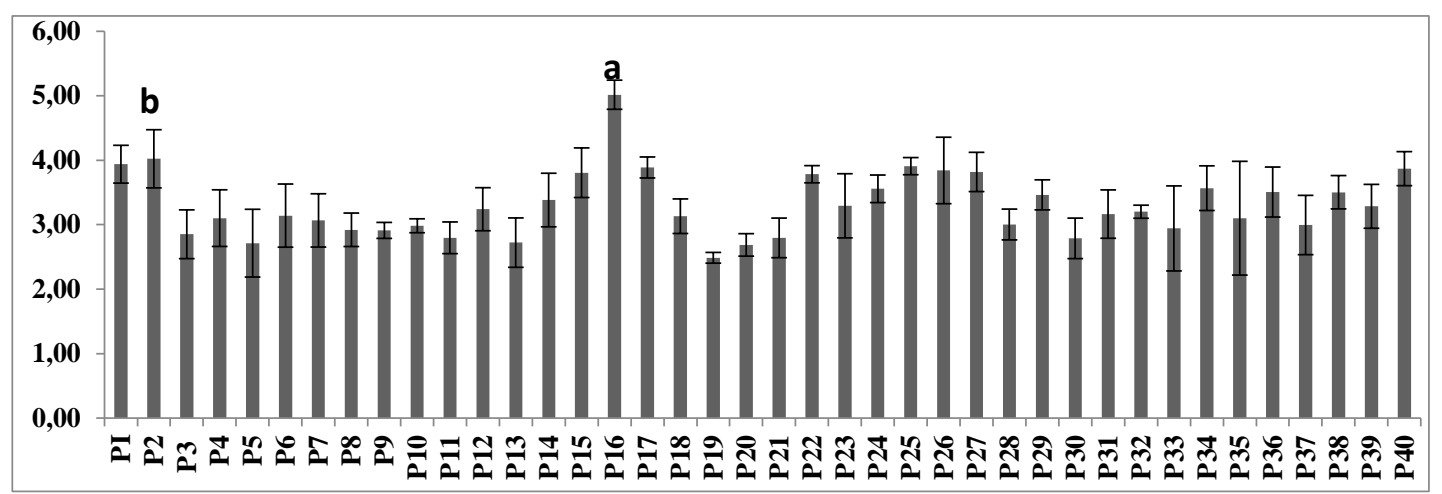

Figure 3. Mean value of dry weight biomass of 40 populations of Aegilops tauschii. Bar indicating with letters showed variation. While other populations showed a close relationship with each other

Table 4. Allele amplification, allele frequency, and polymorphism information content for used SSR primers in 40 Aegilops tauschii populations

\begin{tabular}{c|c|c|c}
\hline Primers & $\begin{array}{c}\text { Allele } \\
\text { Amplification }\end{array}$ & Allele Frequency & PIC Value \\
\hline AG-15 & 7 & 0.82 & 0.30 \\
AG-7 & 4 & 0.41 & 0.63 \\
AG-18 & 2 & 1.00 & 0.00 \\
AG-14 & 4 & 1.00 & 0.53 \\
AG-20 & 2 & 1.00 & 0.00 \\
AG-19 & 4 & 0.87 & 0.21 \\
AG-12 & 2 & 1.00 & 0.00 \\
AG-5 & 2 & 1.00 & 0.00 \\
\hline Mean & 3.37 & 0.88 & 0.20 \\
\hline
\end{tabular}

On the base of cluster analysis (Fig. 4), 40 populations of Ae. tauschii were divided into three groups (I-III). Furthermore, the genetic relationships between populations elucidated by a dendrogram. Group I has six populations from Shanxi, Henan, and 
Shandong, showing their similar genetic background. Group II clusters have 26 populations of Ae. tauschii from Shaanxi, Shandong, Hebei, Henan, and Shandong provinces. The populations collected from Shaanxi, Henan, Shanxi, and Shandong were present in-group III. These results illustrated that the populations in the same group have a similar genetic background.

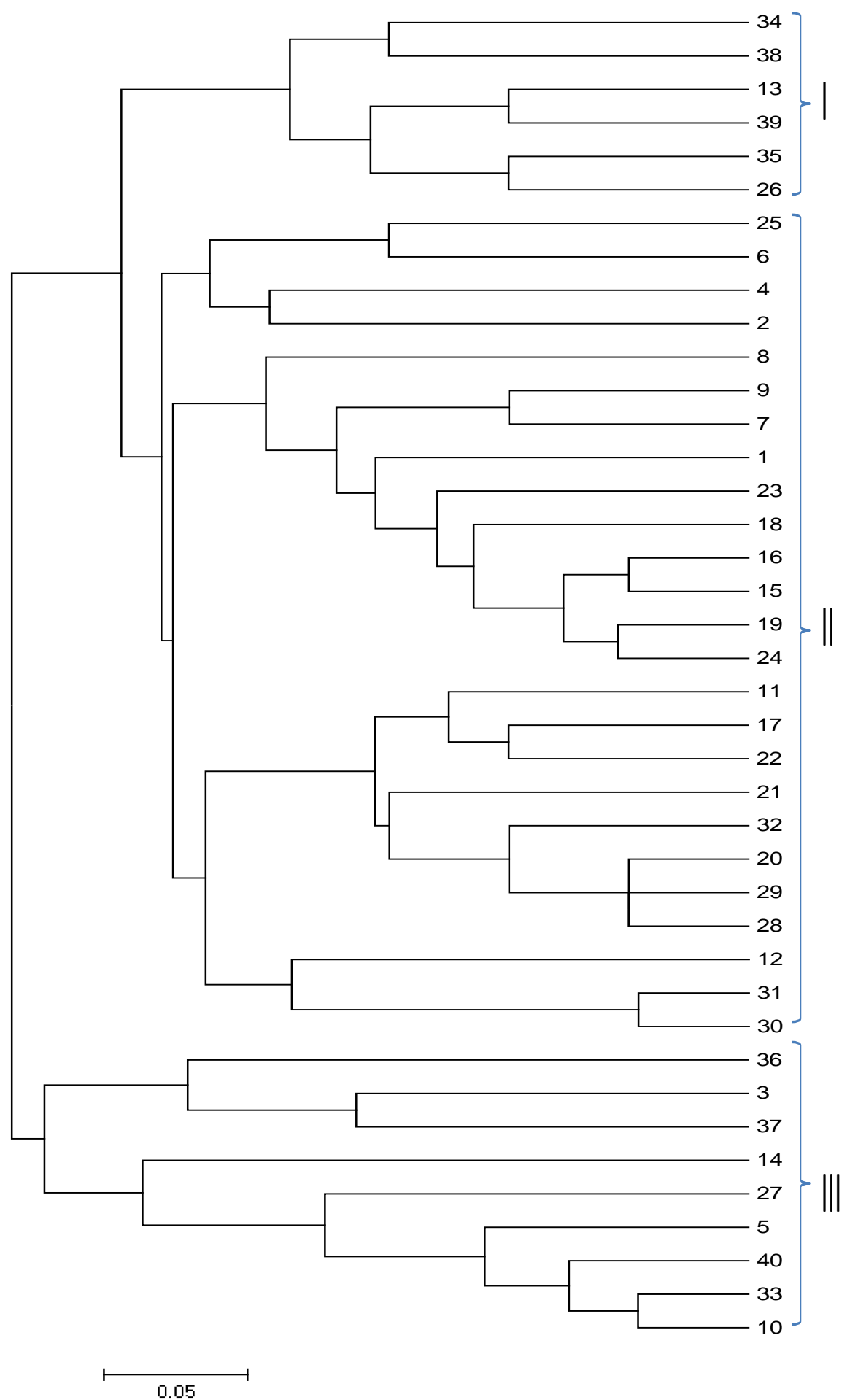

Figure 4. Genetic relationship determined by PowerMarker (MEGA 3.5) cluster analysis based on the similarity coefficient of 40 populations of Ae. tauschii. 


\section{Discussions}

The results showed the significant effect of Ae. tauschii on the genetic diversity by using morphysiological traits and SSR markers. Fifty-five populations of Ae. tauschii showed variances on the base of the morphysiological characteristics (Naghavi et al., 2007). Based on data recording plant height, some populations showed a close relationship with each other while populations 3 and 15 showed variation. Zara et al. (2010) and Ojaghi et al. (2010) reported high diversity in Ae.tauschii on the base of plant height, which is a little larger than ours. In our results, dry weight biomass showed close relationships between populations while in some populations showed variations. Zaharieva et al. (2003) reported higher genetic diversity in three species of Aegilops on the base of different morphological traits. Agronomic traits are available for wheat improvement and showed a high level of genetic diversity (Giuliani et al., 2009). Knaggs et al. (2000) reported that morphysiological characteristics of Ae. tauschii could be used in crop improvement varieties. Similarly, in the case of many studies, they believe that seed collection belongs to geographic regions in morphological diversity. Many studies showed that morphological and physiological parameters are not in compliance with the genetic diversity, while molecular markers cover a large part of the genome including coding and noncoding parts (Semagn, 2002). Our results showed that on the base of the molecular marker relationship between Ae. tauschii was obvious, while the genetic difference is present.

Genetic diversity based on morphological and physiological parameters were not confirming like SSR markers. No relationship was present between morphological and physiological parameters with genetic diversity (Tahernezhad et al., 2010). In microsatellite markers, simple sequence repeats (SSR) revealed higher genetic diversity in populations. A high level of polymorphisms and average allelic richness showed a high level of genetic diversity in crops. SSR markers also confirmed the phenotypic evaluation of populations (Zaharieva et al., 2003). The result on the base of eight SSR primers and morphological traits showed genetic diversity between populations. Obtained results were confirmed by Naghavi et al. (2007) with a PIC value of 9.21 and an allelic range of 6-15 that was achieved by the SSR marker and it could be helpful for a breeder in a crop breeding program. Results on the base of eight primers showed genetic diversity between populations. In the evaluation of the genetic diversity in Iranian Ae. tauschii populations using 13 microsatellites, a total of 66 alleles were amplified with PIC value of 0.65 (Saeidi et al., 2006). In our study, maximum (PIC) was 0.63 (Table 4) with the mean of 0.20 , that was lower than 0.82 (Naghavi et al., 2007). Similarly, the genetic diversity of 46 Commelina communis populations using 12 SSR markers were assessed with an average PIC value of 0.20 (Yang et al., 2018). PIC values showed variation because they depended on (GT) content, number allele per locus, and type of motifs (Roder et al., 1995). Amplification of wheat SSR primers in Ae. tauschii (DD) showed similar regions between Ae. tauschii and wheat genome (Zhang et al., 2004). Microsatellite markers from the D genome revealed allelic amplification which showed polymorphism in Ae. tauschii populations of China. Allelic amplification showed a high level of genetic diversity in Ae. tauschii populations of Iran (Saeidi et al., 2006). This showed the relationship between populations and subdivisions in groups showed diversity. Amplification of universal wheat primers in Ae. tauschii of China showed the close relationship between wheat and Ae. tauschii (Pestsova et al., 2000).

Bibi et al. (2009) and Akhundova (2010) reported genetic variation among wheat populations using microsatellite markers with an allelic range of 2-4. SSR markers in 
wheat populations were reported with 3.6 alleles per primer (Ahmad, 2002; AlmanzaPinzon et al., 2003). Genetic diversity among wheat populations were studied with 21 SSR markers, which had an allelic range of 2-6, genetic similarity coefficient variation ranged from 0.45-0.90, and an average PIC value was 0.47 (Singh et al., 2006). Cluster analysis showed that populations from the same geographical region were present in the same group and their division in groups showed diversity. Populations from the same region have a great chance of being descended from a similar ancestor. On the basis of the genetic similarity coefficient, Ae. tauschii was divided into different groups. Cluster analysis divided Ae. tauschii (Iranian populations) into different groups and reported a high level of genetic variation between populations (Zahra et al., 2010).

\section{Conclusion}

Studying of Ae. tauschii populations of China by using molecular markers and morphysiological parameters has an essential role in wheat crop improvement. It is evident through molecular marker that Ae. tauschii will be helpful for crop breeding programs. Cluster analysis divided Ae. tauschii populations into three groups, which showed diversity and its different origins. High PIC value, allele amplifications and major allele frequency in some primers showed that specific parts of Ae. tauschii could be a valuable source for wheat crop improvement. Due to a rich source of potential variability, Ae. tauschii may perform vary when herbicides are applied in different wheat-growing areas. The results obtained from the study will help to weed management strategies in these regions.

Ethics and conflict of interests. The authors declare compliance with ethical standards and that they have no conflict of interests.

Acknowledgments. This study was funded by the National Key Research and Development Program of China (2016YFD0300701).

\section{REFERENCES}

[1] Ahmad, M. (2002): Assessment of genomic diversity among wheat genotypes as determined by simple sequence repeats. - Genome 45: 646-651.

[2] Almanza-Pinzon, M. I., Khairallah, M., Fox, P. N., Warburton, M. L. (2003): Comparison of molecular markers and coefficients of parentage for the analysis of genetic diversity among spring bread wheat accessions. - Euphytica 130: 77-86.

[3] Bibi, S., Dahot, M. U., Khan, I. A., Khatri, A., Naqvi, M. H. (2009): Study of genetic diversity in wheat (Triticum aestivum L.) using random amplified polymorphic DNA (RAPD) markers. - Pakistan Journal of Botany 41: 1023-1027.

[4] Dudnikov, A. J. (2000): Multivariate analysis of genetic variation in Aegilops tauschii from the world germplasm collection. - Genetic Resources and Crop Evolution 47: 185-190.

[5] Giuliani, A., Karagoz, A., Zencirci, N. (2009): Emmer (Triticum dicoccon) production and market potential in marginal mountainous areas of Turkey. - Mountain Research and Development 29: 220-229.

[6] Hoagland, D. R., Arnon, D. I. (1950): The water-culture method for growing plants without soil. - California Agricultural Experiment Station 147: 32-37.

[7] Hsam, S. L. K., Kieffer, R., Zeller, F. J. (2001): Significance of Aegilops tauschii glutenin genes on bread-making properties of wheat. - Cereal Chemistry 78: 521-525. 
[8] Kalia, B., Wilson, D. L., Bowden, R. L., Singh, R. P., Gill, B. S. (2016): Adult plant resistance to Puccinia triticina in a geographically diverse collection of Aegilops tauschii. - Genetic Resources and Crop Evolution 64: 913-926.

[9] Knaggs, P., Ambrose, M. J., Reader, S. M., Miller, T. E. (2000): Morphological characterization and evaluation of the subdivision of Aegilops tauschii Coss. - Wheat Information Service 91: 15-19.

[10] Li, S. C., Mo, D. W. (2004): Textual study on Carbonized wheat in the Donghui Mountain site. - Archaeological Science Reports 6: 51-60.

[11] Li, M. W. (2005): Review of the 100 years' studies of the Silk Road. - Northwest Ethnonational Studies 2: 90-106.

[12] Majidi, M. M., Mirlohi, A., Amini, F. (2009): Genetic variation, heritability, and correlations of agro-morphological traits in tall fescue (Festucaanndinacea Schreb). Euphytica 43: 323-331.

[13] Mian, M. A., Saha, M. C., Hopkins, A. A., Wang, Z. Y. (2005): Use of tall fescue ESTSSR markers in phylogenetic analysis of cool-season forage grasses. - Genome 48: 637647.

[14] Naghavi, M. R., Mardi, M., Pirseyedi, S. M., Kazemi, M., Potki, P., Ghaffari, M. R. (2007): Comparison of genetic variation among accessions of Aegilops tauschii using AFLP and SSR markers. - Genetic Resources and Crop Evolution 54: 237-240.

[15] Ogbonnaya, F. C., Halloram, G. M., Lagudah, E. S. (2005): D genome of wheat-60 years on from Kihara, Sears, and McFadden. - In: Tsunewaki, K. (ed.) Frontiers of Wheat Bioscience, the 100th Memorial Issue of Wheat Information Service. Yokohama: Kihara Memorial Yokohama Foundation for the Advancement of Life Sciences, pp. 205-220.

[16] Ojaghi, J., Akhundova, E. (2010): Genetic diversity in doubled haploids wheat based on morphological traits, gliadin protein patterns, and RAPD markers. - African Journal of Agriculture and Research 5: 1701-1712.

[17] Pestsova, E., Korzun, V., Goncharov, N. P., Hammer, K., Ganal, M. W., Roder, M. S. (2000): Microsatellite analysis of Aegilops tauschii germplasm. - Theory of Applied and Genetics 101: 100-106.

[18] Roder, M. S., Plaschke, J., Konig, S. U., Borner, A., Sorrells, M. E. Tanksley, S. D., Ganal, M. W. (1995): Abundance, variability and chromosomal location of microsatellites in wheat. - Molecular Genetics and Genomics 246: 327-333.

[19] Roy, A., Bandyopadhyay, A., Mahapatra, A. K., Ghosh, S. K., Singh, N. K., Bansal, K. C., Koundal, K. R., Mohapatra, T. (2006): Evaluation of genetic diversity in jute (Corchorus species) using STMS, ISSR, and RAPD markers. - Plant Breeding 125: 292-297.

[20] Saeidi, H., Rahiminejad, M. R., Vallian, S., Heslop-Harison, J. S. (2006): Biodiversity of diploid D-genome Aegilops tauschii Coss. in Iran measured using microsatellites. - Genetic Resources and Crop Evolution 53: 1477-1484.

[21] Semagn, K. (2002): Genetic relationships among ten ended types as revealed by a combination of morphological, RAPD and AFLP markers. - Hereditas 137: 149-156.

[22] Shah, Z. H., Munir, M., Kazi, A. M., Mujtaba, T. (2009): Molecular markers based identification of diversity for drought tolerance in bread wheat varieties and synthetic hexaploid. - Current Issues in Molecular Biology 11: 101-110.

[23] Singh, R., Kumar, N., Bandopadhyay, R., Rustgi, S., Sharma, S., Balyan, H. S., Gupta, P. K. (2006): Development and use of anchored-SSRs to study DNA polymorphism in bread wheat (Triticum aestivum L.). - Molecular Ecology Resources 6: 296-299.

[24] Sun, X., Hu, L. X., Xie, Y., Fu, J. (2014): Evaluation of genotypic variation in heat tolerance of tall fescue by functional traits. - Euphytica 199: 247-260.

[25] Tahernezhad, Z., Zamani, M. J., Solouki, M., Zahravi, M., Imamjomeh, A. A., Jafaraghaei, M., Bihamta, M. R. (2010): Genetic diversity of Iranian Aegilops tauschii Coss. using microsatellite molecular markers and morphological traits. - Molecular Biology Reports 37: 3413-3420. 
[26] Vieira, M. L. C., Santini, L., Diniz, A. L., Munhoz, C. de F. (2016): Microsatellite markers: what they mean and why they are so useful. Genetics and Molecular Biology 39: 312-328.

[27] Wang, J., Luo, M. C., Chen, Z., You, F. M., Wei, Y., Zheng, Y. (2013): Aegilops tauschii single nucleotide polymorphisms shed light on the origins of wheat D-genome genetic diversity and pinpoint the geographic origin of hexaploid wheat. - New Phytologist 198: 925-937.

[28] Wei, H., Li, J., Peng, Z., Lu, B., Zhao, Z., Yang, W. (2008): Relationships of Aegilops tauschii revealed by DNA fingerprints: The evidence for agriculture exchange between China and the West. - Progress in Natural Sciences 18: 1525-1531.

[29] Yang, J., Yu, H. Y., Li, X. J., Dong, J. (2018): Genetic diversity and population structure of Commelina communis in China based on simple sequence repeat markers. - Journal of Integrative Agriculture 17: 2292-2301.

[30] Zaharieva, M., Dimov, A., Stankova, P., David, J., Monnereux, P. (2003): Morphological diversity and potential interest for wheat improvement of three Aegilops tauschii L. species from Bulgaria. - Genetic Resources and Crop Evolution 50: 507-517.

[31] Zahra, T., Mohammad, J. Z., Mahmood, S., Mehdi, Z., Abbas, A. I., Mohammad, J., Mohammad, R. B. (2010): Genetic diversity of Iranian Aegilops tauschii Coss. by using microsatellite molecular markers and morphological traits. - Molecular Biology Reports 37: 3413-3420.

[32] Zhang, L. Q., Liu, D. C., Yan, Z. H., Lan, X. J., Zheng, Y. L., Zhou, Y. H. (2004): Rapid changes of microsatellite flanking sequence in the allopolyploidization of new synthesized hexaploid wheat. - Science in China 7: 553-561.

[33] Zhang, C. X., Li, X. J., Huang, H. J., Wei, S. H. (2007): Alert and prevention of the spreading of Aegilops tauschii, the worst weed in a wheat field. - Acta Phytophylacica Sinica 34: 103-106.

[34] Zhao, J., Ren, W., Zhi, D., Wang, L., Xia, G. (2007): Arabidopsis DREB1A/CBF3 bestowed transgenic tall rescue increased tolerance to drought stress. - Plant Cell Reports 26: $1521-152$. 